\title{
Interfaces
}

\section{Voir et dessiner la Grande Guerre : carnets d'artistes combattants}

\section{Marco Falceri}

\section{(2) OpenEdition}

1 Journals

Édition électronique

URL : https://journals.openedition.org/interfaces/2528

DOI : 10.4000/interfaces.2528

ISSN : 2647-6754

Éditeur :

Université de Bourgogne, Université de Paris, College of the Holy Cross

\section{Édition imprimée}

Date de publication : 12 juillet 2021

ISSN : 1164-6225

\section{Référence électronique}

Marco Falceri, « Voir et dessiner la Grande Guerre : carnets d'artistes combattants », Interfaces [En ligne], 45 | 2021, mis en ligne le 12 juillet 2021, consulté le 15 septembre 2021. URL : http:// journals.openedition.org/interfaces/2528; DOI : https://doi.org/10.4000/interfaces.2528

Ce document a été généré automatiquement le 15 septembre 2021.

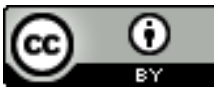

Les contenus de la revue Interfaces sont mis à disposition selon les termes de la Licence Creative Commons Attribution 4.0 International. 


\title{
Voir et dessiner la Grande Guerre : carnets d'artistes combattants
}

\author{
Marco Falceri
}

1 Les carnets d'artistes combattants de la Grande Guerre constituent des productions artistiques et documentaires restituant les expériences visuelles, sociales et émotionnelles des témoins des combats : artistes professionnels surtout (peintres, sculpteurs, graphistes et architectes), mais aussi artistes-écrivains et créateurs nonprofessionnels qui adoptent également le format du carnet pour dessiner la guerre dans les intervalles d'inaction ou de retour au foyer. Les non-combattants proviennent de la société civile ou du domaine militaire, alors que les combattants ne dépendent que des unités combattantes des armées. De surcroît, le qualificatif lexical "combattant » n'implique pas l'utilisation des armes, ni une apologie du métier de soldat, mais évoque plutôt l'expérience concrète de chacun face aux «dangers " du front (Cru 23-24 ; Keegan 286-92) ${ }^{1}$.

Il s'agira ici d'analyser les carnets de guerre en tant qu'objets visuels et testimoniaux, à l'aune des expériences d'artistes combattants tels que Max Beckmann, Henri Camus, Joseph Dezitter, André Mare, André Masson et Adrien Ouvrier. En premier lieu, le format du carnet sera confronté aux collections numériques et aux expositions virtuelles du centenaire de la Grande Guerre, selon une démarche qui vise à décrypter ses spécificités techniques et matérielles par rapport au format éditorial de l'album. Lorsque les artistes combattants reproduisent leurs témoignages iconographiques dans les albums d'estampes, il importe de ne pas superposer les deux termes spécifiques, car le carnet de croquis constitue un support pour l'expérimentation artistique et non pas pour la conservation ou la reproduction d'images. L'enquête se concentrera ensuite sur les pratiques d'édition et d'exposition du carnet de guerre en adoptant une approche diachronique et socio-historique. Enfin, il s'agira d'évaluer tout aussi bien les fonctions iconographiques que les usages expérimentaux ou pragmatiques des carnets d'artistes combattants, au travers de diverses pratiques et expériences. 


\section{Albums ou carnets de guerre ? Enjeux philologiques et patrimoniaux}

3 L'utilisation des carnets d'artistes est largement attestée depuis la Renaissance européenne et s'intensifie à l'ère contemporaine, marquée par l'industrialisation de la papeterie et par la numérisation des artefacts. Ainsi les éditions des carnets d'études de Léonard de Vinci ou d'Eugène Delacroix ont désormais une histoire pluriséculaire qui comporte la prise en compte de l'intermédialité. Ces productions documentaires, tout à la fois artistiques et testimoniales, ont fait l'objet d'éditions livresques, d'expositions rétrospectives, enfin d'opérations de conservation et de valorisation du patrimoine par la mise en ligne d'éditions numériques sous ou sans licence ouverte ${ }^{2}$. En ce qui concerne les carnets de guerre, leurs origines incertaines remontent plausiblement à la deuxième moitié du $\mathrm{XIX}^{\mathrm{e}}$ siècle. Charles Baudelaire commente les témoignages graphiques du "soldat artiste " Constantin Guys vers 1862 (Montier 187) mais l'historiographie relève la diffusion massive des carnets de combattants dans la Grande Guerre, en valorisant l'iconographie artistique ou les pratiques du journal intime : « [...] comme la correspondance, le carnet était un fragment de vie personnelle et familiale » (Cazals et Rousseau 30) ${ }^{3}$.

4 Les carnets d'artistes combattants figurent dans les corpus de l'édition d'art et dans ceux concernés par la mise en ligne de collections numériques. En France, dans le cadre des missions pédagogiques du centenaire de la Grande Guerre, les carnets d'anciens combattants ont fait l'objet de la Grande Collecte patrimoniale, privilégiant surtout le modèle littéraire du carnet de route ou du journal intime, qui demeurent les plus nombreux et sont disséminés aussi bien dans les collections privées que dans les " collections de guerre » des bibliothèques (Didier 133-49)4. Les chercheurs constatent les avantages et les limites des pratiques documentaires dans les ressources du numérique. Si les sites internet fournissent une description détaillée des collections, les notices bibliographiques ne spécifient cependant pas toujours à quelle date ou à quel endroit les images et/ou les textes numérisés ont été mis en ligne. L'absence de la date constitue un obstacle pour la recherche, de manière tout à fait analogue au défaut de la signature. La diversité des pratiques et des acteurs pose la question du formatage visuel des documents dans l'écosystème médial et politique d'internet.

5 Il faut considérer les artefacts informatiques en tant que produits d'une action collective mise à disposition des usagers. De plus, les collections numérisées et les expositions virtuelles nécessitent une maîtrise de la pratique philologique et une démarche sélective à l'égard des interfaces linguistiques et des médias. À l'aide des ressources informatiques, il importe d'examiner les indices matériels et les filiations généalogiques inhérents aux processus de transmission documentaire, en considérant les questions conjointes de l'anonymat et de l'auctorialité, du cadre d'analyse et de la condition d'accessibilité aux sources.

6 À l'occasion de la commémoration du centenaire de la Grande Guerre, les carnets d'artistes combattants ont été présentés dans quelques expositions virtuelles à dispositif immersif, et soumis non plus seulement au " regard des spécialistes " (Heinich 9), mais au public bien plus hétérogène des internautes. Par l'intermédiaire de l'IMEC (Institut Mémoires de l'Édition Contemporaine) et de La Contemporaine (Bibliothèque, archives, musée des mondes contemporains), ces objets ont acquis une nouvelle visibilité médiatique, qui permet de les exploiter sans recourir nécessairement 
aux collections imprimées ou manuscrites. Les expositions virtuelles de l'IMEC ( Grande Guerre en archives ») 5 et de La Contemporaine ("Camus, Krier, Lotze : itinéraires croisés de trois artistes combattants $)^{6}$ valorisent respectivement les carnets de guerre de l'artilleur et camoufleur André Mare, et ceux du fantassin et brancardier Henri Camus. En dépit de leur diversité, ces objets d'art et d'histoire n'ont pas obtenu un égal traitement bibliographique. Si la quinzaine de pages extraites des carnets reliés de Mare ne sont reproductibles ou téléchargeables que sous autorisation (Fig. 1), en revanche, plus de deux cent feuilles issues des carnets déliés et dispersés de Camus sont intégralement consultables et visualisables, bien qu'utilisables en tant que reproductions photographiques sous droits réservés (Fig. 2).

Figure 1. André Mare, Le canon de 280 camouflé, 1915. Aquarelle et encre sur papier, dimensions inconnues (carnet de guerre $\mathrm{n}^{\circ} 2$ ). Institut Mémoires de l'Édition Contemporaine, Saint-Germain-laBlanche-Herbe.

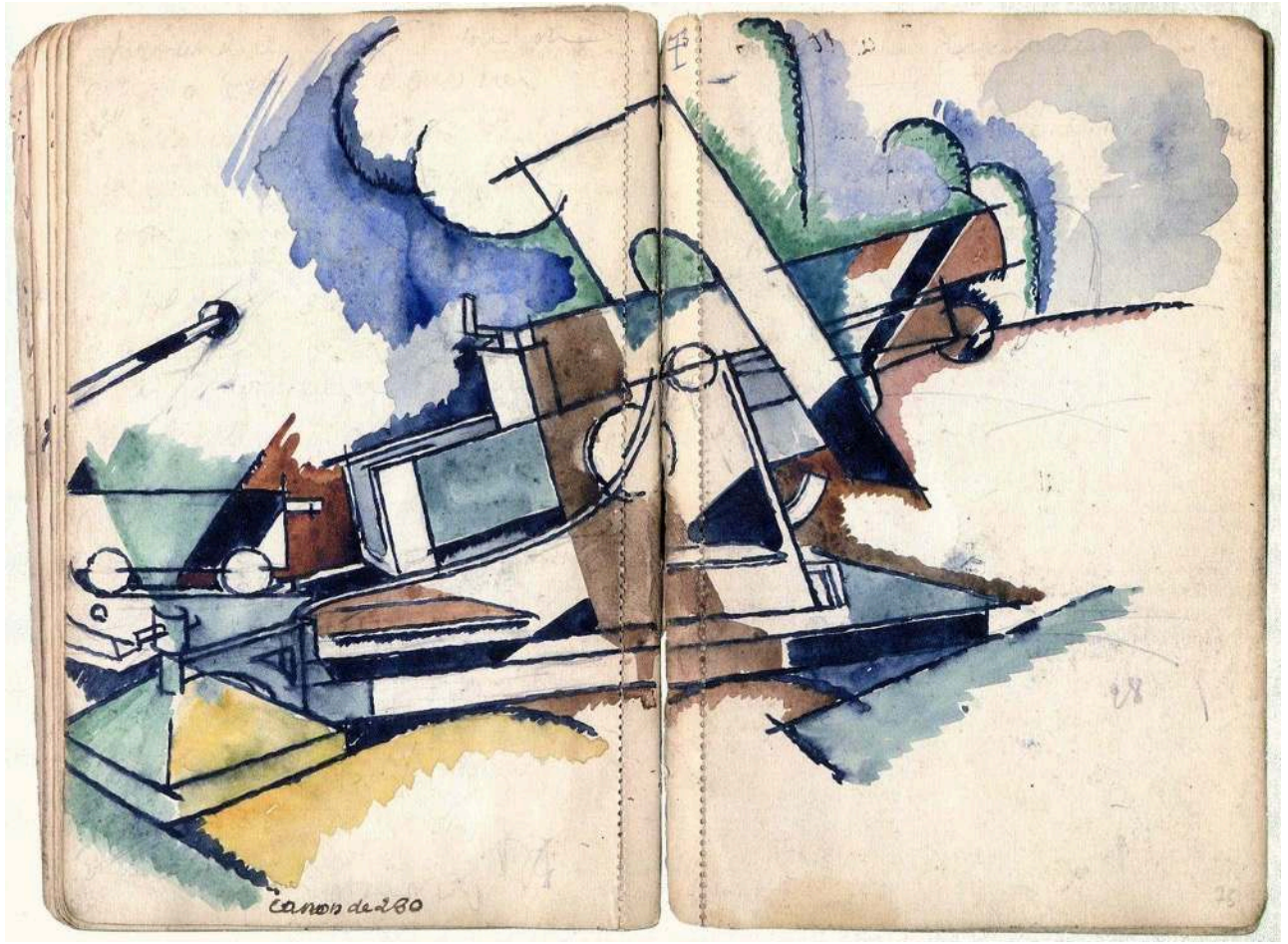

(c) https://upload.wikimedia.org/wikipedia/commons/4/41/

André_Mare_1885-1932_Camouflaged_280_Gun_sketch_in_ink_and_watercolour.jpg

Figure 2. Henri Camus, Une corvée revenant du travail de nuit, 1918. Aquarelle, crayon et encre sur papier, dimensions inconnues. Collection La contemporaine, Nanterre.

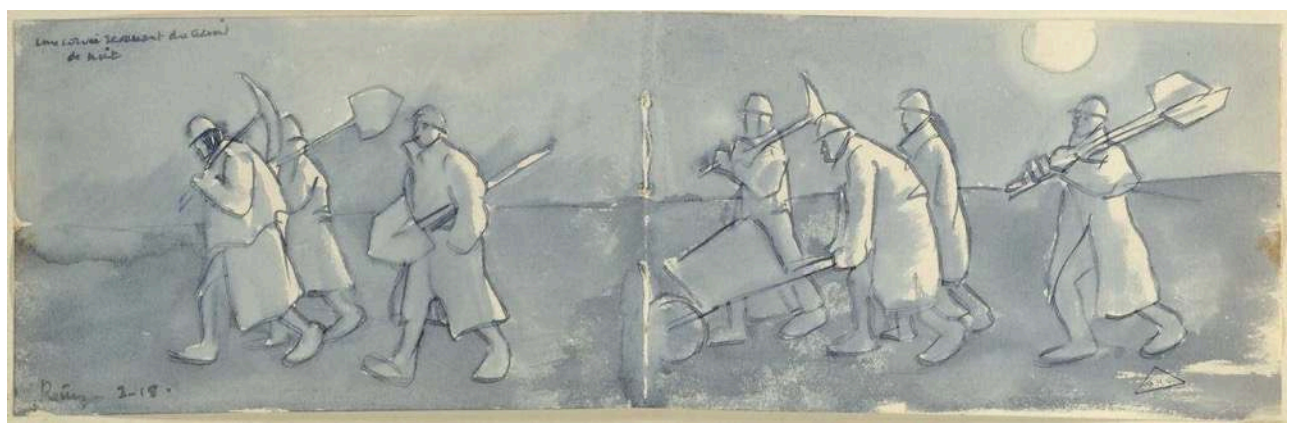

(c) Henri Camus. 
7 D'emblée, il faut se demander s'il s'agit d'une donation patrimoniale (fonds Camus), d'un achat de collection ou d'un dépôt d'archive (fonds Mare). Cette disparité dans la distribution des fonds ne découle pas toujours des "droits d'auteur ", mais aussi des enjeux politiques et mémoriaux autour de l'œuvre d'art et de la renommée de l'artiste combattant. Les carnets de Mare, peintre et architecte cubiste, ont précédemment fait l'objet d'une édition scientifique (Graffin), d'un catalogue d'exposition sur les rapports entre avant-garde cubiste et camouflage militaire (Zapata-Aubé), ainsi que d'une docufiction cinématographique (Tonolli). Au contraire, ceux d'un créateur nonprofessionnel tel que Camus ont connu une première réévaluation dans l'exposition $\mathrm{Vu}$ du front. Représenter la Grande Guerre organisée en 2014 à Paris au Musée de l'Armée, lorsque certains croquis du front ont été exposés avec une partie de la correspondance de guerre (Musée de l'Armée et Bibliothèque Documentation Internationale Contemporaine $94 ; 100)$ :

Appartenant à la classe 14, Camus est mobilisé dès le début de la guerre. Il n'a pas de formation artistique, mais pratique assidûment le dessin depuis l'enfance et continue pendant toute la durée du conflit (il subsiste plusieurs centaines de croquis et aquarelles). [...] Une quinzaine de ses dessins sont acquis auprès de lui dès le printemps 1919 par le musée de la Guerre, puis plus de deux cent cinquante donnés par sa famille en 2008. (264)

8 À l'été 1914, si Camus n'a pas encore entrepris une carrière artistique, son témoignage combattant n'est pas moins significatif que celui de Mare. On peut confronter ainsi deux formats documentaires du carnet (relié/délié) et deux profils socioprofessionnels d'artistes combattants (professionnel/non-professionnel). Conjointement à l'exposition commémorative et documentaire du Musée de l'Armée, l'exposition virtuelle de La Contemporaine valorise le fonds Camus et les productions iconographiques d'artistes combattants français et allemands, mais classe les feuilles éparses des carnets de guerre en " différents formats » sous le genre de l'« album », en brouillant ainsi, dans la même notice, la différence entre les correspondances et les récits personnels ${ }^{7}$. Afin de décrire les supports à dimensions variables du fond (lettres et cartes postales, récits autobiographiques, feuilles collées sur cartons contenant dessins et peintures), la notice reprend - lettre à l'appui envoyée par un ancien combattant à l'artiste et datée du 14 avril 1970 - la notion aussi générique qu'inclusive d'album de guerre : « Je ne veux pas tarder un instant à venir vous remercier de m'avoir confié votre bel album de croquis de 14-18. [...] Quel dommage que les circonstances ne vous aient pas permis, à l'époque, de les publier $! »^{8}$.

9 Bien qu'il n'existe pas encore d'édition monographique, il serait cependant erroné d'affirmer que les croquis et les aquarelles de guerre de Camus demeurent encore inédits. À l'état fragmentaire, les feuilles déliées des carnets se prêtent à la comptabilisation et à la comparaison, à la reconstruction philologique et à l'analyse iconographique, poétique et stylistique, à l'inverse des carnets de Mare, qui demeurent reliés et numérotés par l'artiste combattant. Il faut exercer une critique philologique des artefacts matériels et informatiques, laquelle implique une sérialisation préalable des documents utilisables. Comme en philologie classique, en philologie matérielle ou numérique, on ne remonte pas jusqu'à la forme unique et originelle du document, à l'archétype, mais on récupère et décrit les formats des carnets en termes de modèles, dimensions, proportions et durées. Reliés ou déliés, originaux ou reproduits, les carnets d'artistes combattants doivent être différenciés selon les modalités de conservation et de transmission documentaire (publique/privée), ou encore selon les contextes de 
production et de réception esthétique (synchronique/diachronique). S'agit-il de témoignages de combattants directs ou recomposés ? Constate-t-on des modifications de formats dans les différents processus de création artistique ? Le carnet de croquis se transforme-t-il en album de guerre? Enfin, même si le titre d'un tableau du peintre italien Albano Vitturi suggère la transmission familiale et intergénérationnelle de l'album (Di Genova 503) ${ }^{9}$, les carnets d'artistes combattants n'ont-ils pas fait l'objet d'éditions, expositions et ventes?

\section{Éditions et expositions des carnets de guerre}

En 1917, les professionnels des Beaux-Arts utilisent le terme " album de guerre " pour qualifier une édition commerciale et imprimée de dessins ou gravures. Souvent, ces éditions d'albums reproduisent les pages des carnets de croquis, quelquefois coloriés à l'aquarelle, à la gouache ou au lavis. Dans "Les estampes et la guerre ", un article de la Gazette des Beaux-Arts repris ensuite dans une monographie (Les estampes. Images et affiches de la guerre, 1919), le critique d'art Noël Clément-Janin énumère un vaste répertoire d'albums de guerre, amalgamant les productions d'artistes combattants et non-combattants sous l'étiquette des documentaires : «Les documentaires sont ceux qui retracent ce qu'ils ont vu, sans y ajouter de leur propre fond, ou, du moins, - Soyons prudent ! - sans y trop ajouter " (Clément-Janin 1917, 361). Parmi les artistes combattants recensés, on retrouve André Mare, qui venait de publier un recueil de vingt-quatre lithographies tirées à 120 exemplaires auprès d'un éditeur inconnu (Dessins faits aux armées) ${ }^{10}$. Les estampes s'inspirent en partie de carnets de croquis et Clément-Janin commente ces images en mobilisant les poncifs nationalistes qui décrient le cubisme, considéré par une partie des élites intellectuelles des pays Alliés comme une dégénération stylistique d'origine allemande: "Je ne croyais pas que l'exécution cubiste appartînt au carnet de poche ; mais l'artiste affirme qu'il a fait ses dessins 'aux armées' : nous devons le croire ; et, pour le surplus, nous ne discuterons pas sur le cubisme, qui est moins de l'art que de l'artifice » (367). Bien que les « albums de gravures » recensés figurent en quantité supérieure, le critique d'art considère les « albums de croquis " d'une valeur inférieure, tant par leur qualité esthétique que par leur prix sur le marché. Les reproductions héliographiques permettent d'adapter les carnets d'artistes combattants au format éditorial de l'album de guerre, largement commercialisé dans les anciens pays belligérants.

11 En janvier 1915, l'École Nationale Supérieure des Beaux-Arts et la Fraternité des Artistes publient sous le haut patronage du Président de la République Raymond Poincaré un Album national de la guerre (Bonnat), estampes reproduisant peintures et dessins d'artistes français, où les illustrations patriotiques de Claude Monet et de Paul Signac côtoient celles d'artistes devenus combattants comme George Desvallières et André Devambez. Le 28 août 1916, l'inspecteur général des Beaux-Arts et éditeur Armand Dayot recense l'œuvre visuelle de l'artiste combattant Mathurin Méheut dans l'hebdomadaire L'Illustration: parues en noir et en couleurs, certaines pages des carnets de guerre sont légendées comme " pages d'album » (Dayot n.p.). En règle générale, l'édition et la presse illustrée transfèrent les pages de carnets d'artistes combattants sous le format éditorial de l'album, qui permet une mise en succession des images reproduites, en l'occurrence encadrées et dotées de légendes explicatives. 
12 Du 27 décembre 1916 au 27 février 1917, les ministères des Beaux-Arts et de la Guerre, avec le journal militaire Bulletin des Armées de la République édité par Berger-Levrault, organisent au Jeu de Paume de Paris le Salon des Armées de la République. Pour faire la promotion du salon public, cinq semaines avant le vernissage (22 novembre 1916), le Bulletin publie l'interview anonymée à un étudiant de l'École Nationale Supérieure de Beaux-Arts :

Je suis parti le 2 août 1914 et, dans mon sac, j'emportais, à côté des flanelles maternelles, mon carnet à dessins et mes crayons. Ils ne m'ont pas quitté un jour. En chemin de fer, au cantonnement, en corvée, dans les tranchées même, je les emporte et je crayonne tout ce que je vois à droite, à gauche et en face. J'ai ainsi des centaines et centaines de croquis de paysages, de camarades, notations rapides de choses entrevues, qui sont pour moi d'inoubliables souvenirs (An. «Le 'Salon' des Armées ", 7).

13 En septembre 1916, le journal populaire illustré Lectures pour tous, édité par Hachette \& Cie, relate l'anecdote légendaire de l'architecte combattant Jacques Théodore Jules Lecomte de Nouÿ, sauvé par son carnet de poche lors d'un assaut d'infanterie : « [...] une balle l'ayant frappé en pleine poitrine, il fut sauvé par son carnet qui, traversé presque en entier, détourna la balle vers le bras » (An. « Nos artistes au front », 1816). Si la presse illustrée décrit l'usage du carnet de guerre comme une pratique relevant à la fois de la biographie et de la mythographie, en revanche le Salon des Armées, organisé au Jeu de Paume de Paris, valorise les carnets d'artistes combattants par des dispositifs d'encadrement dans le contexte d'une exposition collective : cadres rectangulaires ou ovales pour exposer les pages ou les doubles pages sous verre ou sous vitrine ; accrochages de cartons, de panneaux et de planches ficelant les feuilles par agrafes, colles, épingles et punaises ; enfin, reproductions héliographiques des originaux vendues en albums d'estampes et de croquis. Une fois de plus, dans la liste du catalogue du Salon des Armées, le carnet apparaît déclassé, mis à l'écart en tant qu'objet de papeterie, au profit du format éditorial de l'album de guerre (An. «Le 'Salon' des Armées ", 1-40). Et pourtant, les témoignages écrits et visuels des artistes combattants confirment l'usage du carnet, tout comme de bloc-notes, calepins, cahiers d'école et agendas militaires. Sans dispositifs d'encadrement, le carnet d'artiste s'avère en effet très peu adaptable à l'exposition, se prêtant davantage à l'édition et plus récemment aux défis de la numérisation.

Tout autant pendant la Grande Guerre que de nos jours, les acteurs du patrimoine culturel privilégient la forme-archive de l'album pour nommer un corpus iconographique de format éditorial, alors que le carnet désigne plutôt un objet de travail, intime et personnel. Les artistes visuels ou les historiens de l'art adoptent le carnet pour l'« exercice de la notation » (Didi-Huberman 2020, 614) graphique ou photographique, mais le terme n'est pas équivalent ou homologue à celui d'album. Si le carnet reste un support matériel pour l'expérimentation, la gestation et le souvenir de l'expérience visuelle, l'album constitue à l'inverse un recueil d'images éditées ou inédites, un classeur individuel ou collectif exécuté ex post, comme dans le cas des albums de photographies, ou des imagiers numériques des ordinateurs et des dispositifs mobiles $^{11}$. La superposition, voire la confusion terminologique entre l'album et le carnet de guerre ne découle pas seulement des collections numérisées ou des expositions virtuelles du centenaire de la Grande Guerre, mais se repère tout aussi bien dans les domaines de la conservation, de l'édition et de l'exposition. 
Ainsi, dans une monographie parue à la suite d'une donation offerte aux Musées de Vienne (Fonds Adrien Ouvrier), parmi les huit carnets reliés du peintre et fantassin Adrien Ouvrier, un seul exemplaire a été nommé " album » et deux carnets de croquis ont été classés sous "statut différent " puisque provenant d'une collection privée (Lauxerois 17-18). À ce corpus de huit carnets datés de 1914 à 1918, il faut ajouter quatre carnets de route rédigés et dessinés entre 1914 et 1919, les correspondances et quelques photographies. L'album en question n'est qu'un carnet de grand format, alors que les cinq carnets de croquis restants présentent des dimensions plus petites (17-19), tout comme les sept carnets qui datent de la période de formation académique de l'artiste (16).

Dans l'étude des carnets d'artistes combattants, il faut faire une distinction entre le format intégral (relié) et fragmentaire (délié) du document, mais il convient de différencier aussi les trois formats des carnets de croquis (sources iconographiques), des carnets de route (sources littéraires conformes au journal intime ou au récit personnel, bien qu'elles puissent contenir des sources non-textuelles) et des albums (photographies ou plus généralement éditions imprimées). Outre ces productions livresques, on retrouve les lettres et les cartes postales parfois illustrées. De plus, il faut compter toute une production d'estampes, peintures, sculptures, récits, etc. Les enjeux pour les recherches et les montages documentaires restent l'hétérogénéité et l'hybridité des formats : un carnet de croquis peut contenir dessins ou aquarelles, mais aussi des notations écrites ou des photographies.

17 L'emploi du terme " album de guerre " peut trouver une double signification: générique, lorsqu'il s'agit de nommer la forme-archive d'un corpus d'images et/ou de textes visualisables selon un dispositif de présentation livresque, cinématique ou informatique ; spécifique, lorsque le format livresque implique le catalogage d'images reproduites. Selon les conventions du champ académique et universitaire, l'album constitue un recueil d'images encadrées et sérialisées. S'impose ainsi une sorte d'hégémonie visuelle de l'album de guerre, en raison de la petitesse et de la fragilité du carnet d'artiste combattant, un objet de papeterie destiné à l'usage quotidien et souvent au démembrement, comme c'est le cas pour les carnets de chèques ou de timbres-poste. L'étymologie néo-latine du mot (lat. quaternum, français méd. quernet) suggère la matérialité du format livresque in-quarto, qui relie huit pages pliées en quatre dont les pontuseaux ${ }^{12}$ sont horizontaux et non verticaux. Avec la modernisation de l'imprimerie, le petit format in-quarto se confond avec l'ordinaire in-folio (quatre pages pliées en deux avec des pontuseaux perpendiculaires) et le grand in-octavo (seize pages pliées en huit avec des pontuseaux perpendiculaires) (Rouveyre 38).

Les formats des carnets de guerre impliquent une description non seulement de la dimension mais aussi de l'organisation des pages, ainsi que des pratiques de lecture qui comportent des enjeux de présentation, de manipulation et d'accès à l'information. Pour en redéfinir le statut ontologique toujours incertain - « ni œuvre, ni hors-œuvre » -, un colloque organisé en février 2011 à l'Université de Lincoln (Grande-Bretagne) a proposé de mener une réflexion interdisciplinaire sur les carnets d'artistes à partir de la notion de " palimpseste » de Gérard Genette, du couple dialectique " actuel/virtuel » de Gilles Deleuze, ou bien des "suppléments (parerga) » de Jacques Derrida (Bartram, El-Bizri et Gittens 2-26). Au tournant de la Grande Guerre, on répertorie une grande diversité de modèles, tailles, reliures et couvertures, mais seuls deux types de carnets sont commercialisés dans les librairies et les papeteries. Les plus recherchés et utilisés 
par les artistes combattants sont les carnets à feuilles détachables, perforés ou segmentées (Fig. 1) ; ensuite, on retrouve également les carnets à feuilles reliées, généralement par agrafes et colles (Fig. 2). En outre, les magasins qui dispensent les fournitures pour les beaux-arts commercialisent des carnets à dessiner dont les feuilles présentent des formats horizontaux ou verticaux, selon les exigences spécifiques des artistes visuels qui pratiquent respectivement les genres iconographiques du paysage et du portrait, sans exclure les hybridations et les ruptures des conventions. Contrairement à l'album - terme qui, selon Georges Didi-Huberman, désigne un livre d'estampes dont les propriétés spécifiques restent " le cadrage, le montage et l'éclairage des œuvres d'art » (2013 25) -, le carnet constitue le format privilégié des artistes combattants, qui exécutent croquis et esquisses, se déplaçant avec ces objets portatifs sur les lignes de front, entre les cantonnements et les tranchées, ou encore travaillent de mémoire et après-coup.

19 Si les artistes combattants créent un album, il s'agit d'un recueil de "vues photographiques "(Struk 21-48) sans quoi il s'agit, le plus souvent, d'une édition livresque reproduisant ou collectant des croquis originaux. Bien qu'il ne faille pas exclure la possibilité que les artistes combattants emploient les albums pour rassembler des images (produites aussi par divers auteurs), le support premier pour voir et dessiner la Grande Guerre reste le carnet de croquis. Dans une lettre envoyée à sa femme Marguerite Rouja et datée du 9 novembre 1918, le peintre et officier d'infanterie Mathurin Méheut déclare: " Je ne m'attendais pas à être témoin d'un des plus grands spectacles du monde. J'en suis encore bouleversé, enfin on aura les preuves sous formes de croquis et de notes " (Jude et Jude 134). Et le mitrailleur Otto Dix suggère, dans une lettre envoyée à son amie Helene Jacob et datée du 7 janvier 1916 : " $\mathrm{Au}$ prochain salaire que je recevrai, je vous envoie 1 Mark pour m'acheter et m'envoyer un carnet de croquis. Le modèle vous le connaissez déjà » (Dix 440-41) ${ }^{13}$.

\section{Usages pragmatiques et poétiques : expérimentations et mémoires}

Au lieu des supports formatés comme les agendas militaires ou les cahiers d'école, les artistes combattants adoptent les carnets pour dessiner la guerre à l'aide d'outils portatifs comme les crayons et les gommes, les fusains et les pastels, les plumes stylographiques et les encres, les pinceaux et les boîtes d'aquarelles, voire les couleurs en tubes. Une lettre de l'artiste-écrivain Jacques Vaché envoyée à son père James Samuel Vaché et datée du 5 septembre 1915 se termine ainsi : «Post scriptum : vite aussi un bloc pratique de papier à dessin, un bon crayon, gomme, et une petite boite d'aquarelle, avec couleurs en tube » (n.p. lettre $\left.n^{\circ} 16\right)$. En outre, les artistes combattants insèrent dans les carnets cartes et coupures de journaux, photographies ou souvenirs. Mais surtout, comme l'infirmier du front Max Beckmann, ils expérimentent la notation graphique en vue de la mise en œuvre, passant du dessin à la gravure, parfois à la peinture ou à la sculpture (Fig. 3 et 4). 
Figure 3. Max Beckmann, Bombardement aérien, 1915. Encre sur papier, dimensions inconnues. Max Beckmann-Archiv, Munich.

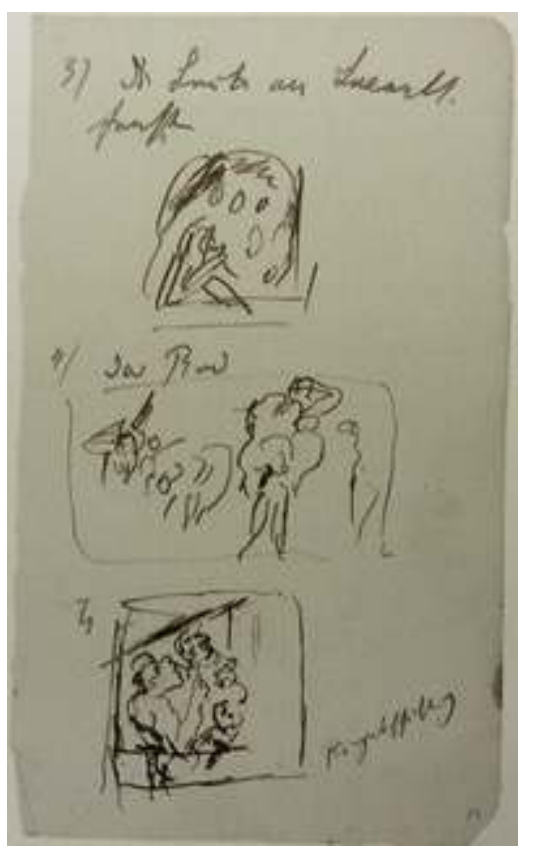

(c) Max Beckmann-Archiv, Munich (cliché-photo Marco Falceri).

Figure 4. Max Beckmann, Attaque aérienne, 1915. Eau-forte, $19.7 \times 14.6 \mathrm{~cm}$. Sprengel Museum, Hanover.

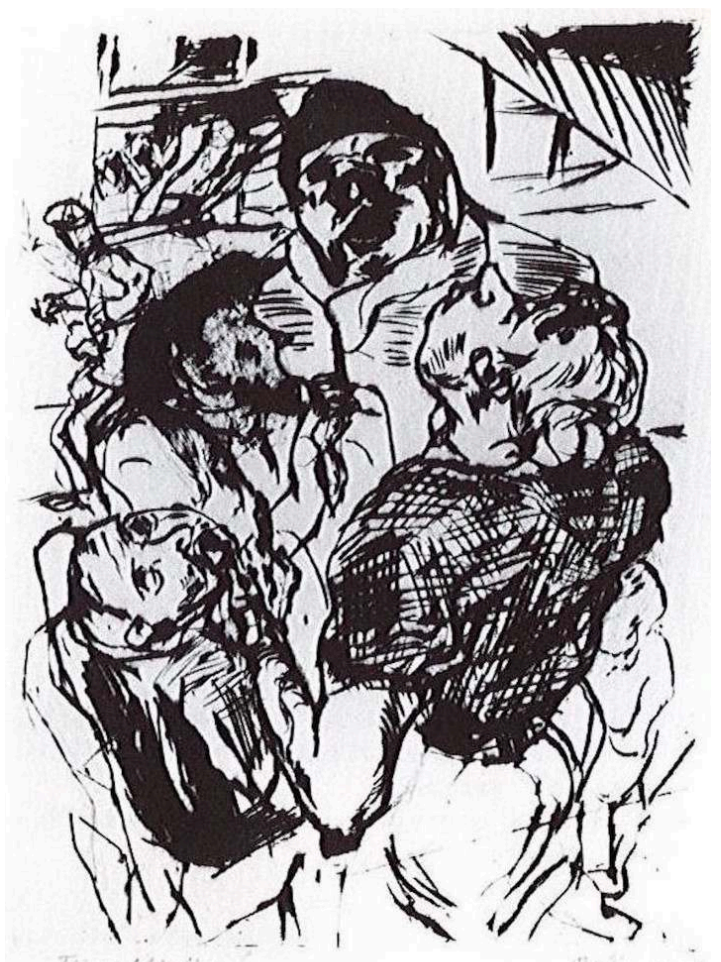

(c) Sprengel Museum, Hanover (cliché-photo Marco Falceri).

21 La pratique du croquis, la notation graphique ou photographique traduisent une expérience visuelle, engageant ainsi une « heuristique du regard » et du « montage » 
(Didi-Huberman 2020, 614 et 621). En outre, comme l'a observé le philosophe Jean-Luc Nancy : « Le mot note - qui renvoie à la notion d'une chose connue, repérée - peut avoir deux valeurs: ou bien celle d'une information fixée, identifiable, ou bien celle d'une ouverture et d'une esquisse en direction de ce qui n'a pas à devenir une information, mais un sens » (32).

Parfois, la notation graphique, chromatique ou photographique implique la restitution de l'œuvre d'art, par-delà tout processus ou procédé de formatage visuel. En histoire de l'art, la méthode génétique ou généalogique commence par les carnets d'artiste pour retrouver les sources de la création ou les motifs de la mise en œuvre. Ainsi, les œuvres d'art et les vies des artistes demeurent mieux connues lorsqu'on dispose de témoignages iconographiques comme les carnets de guerre. La numérisation et la mise en ligne de ces objets d'art et d'histoire représentent un avantage considérable, alors que dans le champ de l'édition livresque les carnets d'artistes ne bénéficient que fort rarement d'une publication optimale en fac-similé et à l'échelle $1: 1$. En outre, à l'instar du découpage et du recadrage d'images photographiques, la reproduction des pages ou des doubles pages des carnets peut s'avérer le produit d'une sélection arbitraire qui comporte une réduction systématique des corpus, à commencer par les pages blanches ou par certains croquis moins lisibles ou déchiffrables. L'édition numérique permet à l'inverse une reproduction optimale et une visualisation des pages aperçues dans leur matérialité stratifiée, recto verso. Malgré les contraintes techniques et juridiques, le numérique permet plus facilement le triage des fichiers contenant les exemplaires reliés ou les feuilles éparses.

Les carnets d'artistes combattants de la Grande Guerre ont fait l'objet d'études et de recherches approfondies, adoptant critères biographiques, chronologiques, topographiques, stylistiques et iconographiques. L'historiographie de l'art prend en compte l'hétérogénéité des corpus documentaires et les régimes de visibilité, en enquêtant aussi sur les instances de la censure et de la propagande. En tant que formats hybrides ou hybridés, les carnets de guerre se distinguent non seulement selon les auteurs, mais aussi selon les usages, les transferts formels et les temporalités. Ils demeurent incontournables pour retracer les rapports et les rencontres des artistes mobilisés au front, en décrivant les trajectoires biographiques et les morphogénèses inhérentes aux processus différenciés de création artistique : changements de format et variations d'échelle, formatages et dé-formatages. Affiches, caricatures, gravures, tableaux, sculptures et créations expérimentales représentant la Grande Guerre impliquent l'étude croisée des carnets, dont les éditions s'avèrent toujours plus nombreuses.

Même si parfois ils possèdent un format fragmentaire, ces objets visuels et testimoniaux déploient leur propre temporalité en fonction des pratiques et des expériences. En dépit de l'immobilisme de la " guerre de tranchée ", les artistes combattants effectuent souvent leurs déplacements de manière autonome, mais surtout selon les ordres des chefs régimentaires recevant les dispositions stratégiques des états-majors. Pour autant, les cartes militaires, les historiques régimentaires et les fiches de mobilisation constituent le complément aussi utile que nécessaire pour l'analyse iconographique de ces productions artistiques et documentaires. Se profile ainsi, dans l'examen attentif des carnets de guerre, tout un potentiel heuristique et documentaire qui suggère une comparaison ontologiquement fondée, mais fort hasardeuse, entre le parcours individuel du combattant et l'itinéraire de voyage d'un 
chroniqueur. Certes, il s'agirait d'un voyage qui n'aurait presque rien en commun avec une escapade touristique, mais d'un trajet qui, au fond, rappelle le chemin descendant que Dante, Virgile et Rimbaud entreprirent dans les cercles de la terre. Anicet aussi, le personnage auquel Louis Aragon fait « regarder le monde au ras du sol » (15) sans accepter les remèdes extrêmes de la folie ou du suicide à la fin de la Grande Guerre, affirme que « [1]a plus belle invention poétique des hommes, c'est l'enfer » (155).

Parmi les notations graphiques, pictographiques ou photographiques des carnets d'artistes combattants, et outre les compositions figuratives et les narrations visuelles qui sont disséminées entre les pages, cadres, inscriptions et maquettes assument une fonction iconographique de premier ordre. Comme on le constate dans une page extraite d'un carnet de Max Beckmann, l'artiste combattant conçoit une série de gravures mettant en séquence des scènes de guerre encadrées et légendées par des inscriptions (Fig. 3 et 4). Traumatisé et démobilisé, depuis 1915, il réalise des gravures et publie, en 1919, deux albums de guerre intitulés Faces et L'Enfer (Ottinger). Ce n'est nullement un cas isolé : les productions ou les éditions des carnets concernent également les artistes décédés pendant la guerre, qui laissent en héritage leur œuvre testamentaire. Il ne faut pas négliger non plus la quantité parfois conséquente de ces objets visuels et testimoniaux qui accompagnent les artistes combattants entre le front et l'arrière, durant et après la guerre : dans l'ensemble, Mare utilise 10 carnets de croquis (Zapata-Aubé 143-45), Ouvrier 8 (Lauxerois) et Beckmann 5 (Zeiller), mais le service armé de ce dernier se termine en mars 1917 ; d'autres, comme le peintre flamand Joseph Dezitter (Fig. 5) ou le surréaliste André Masson (Fig. 6), ne restituent qu'un seul carnet dont les dessins et les aquarelles ont été exécutés de mémoire ${ }^{14}$.

Figure 5. Joseph Dezitter, Le soir de Charleroi - Retraite de Belgique, sans date (1914-1918). Aquarelle, crayon et encre noire sur papier, dimensions inconnues. Comité Flamand de France, Dunkerque.

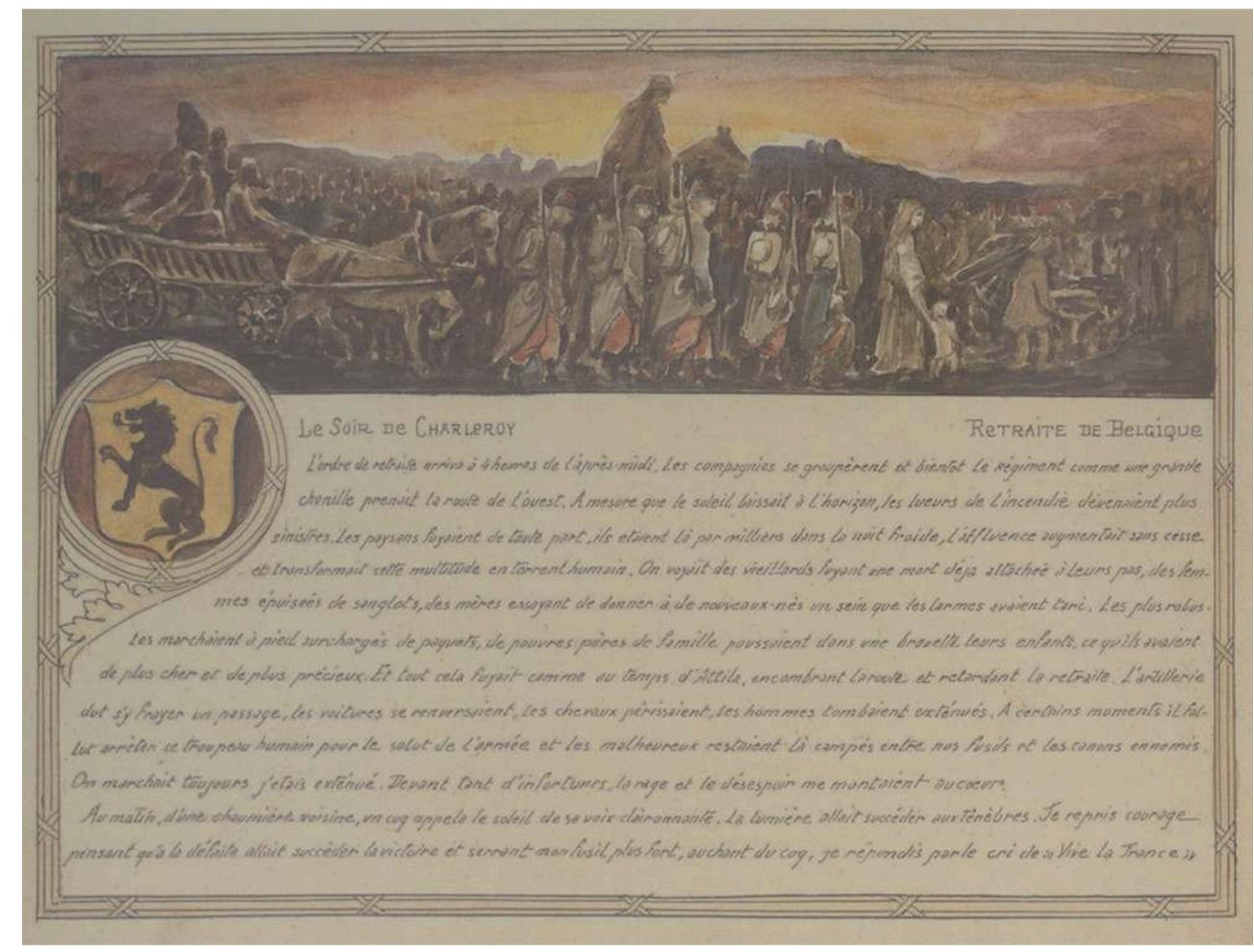

(c)Société Dunkerquoise d'Histoire et d'Archéologie/Michel Tomasek (cliché-photo Marco Falceri). 
Figure 6. André Masson, En 1916, 1971. Feutre sur papier, 21 x30 cm. Collection La contemporaine, Nanterre.

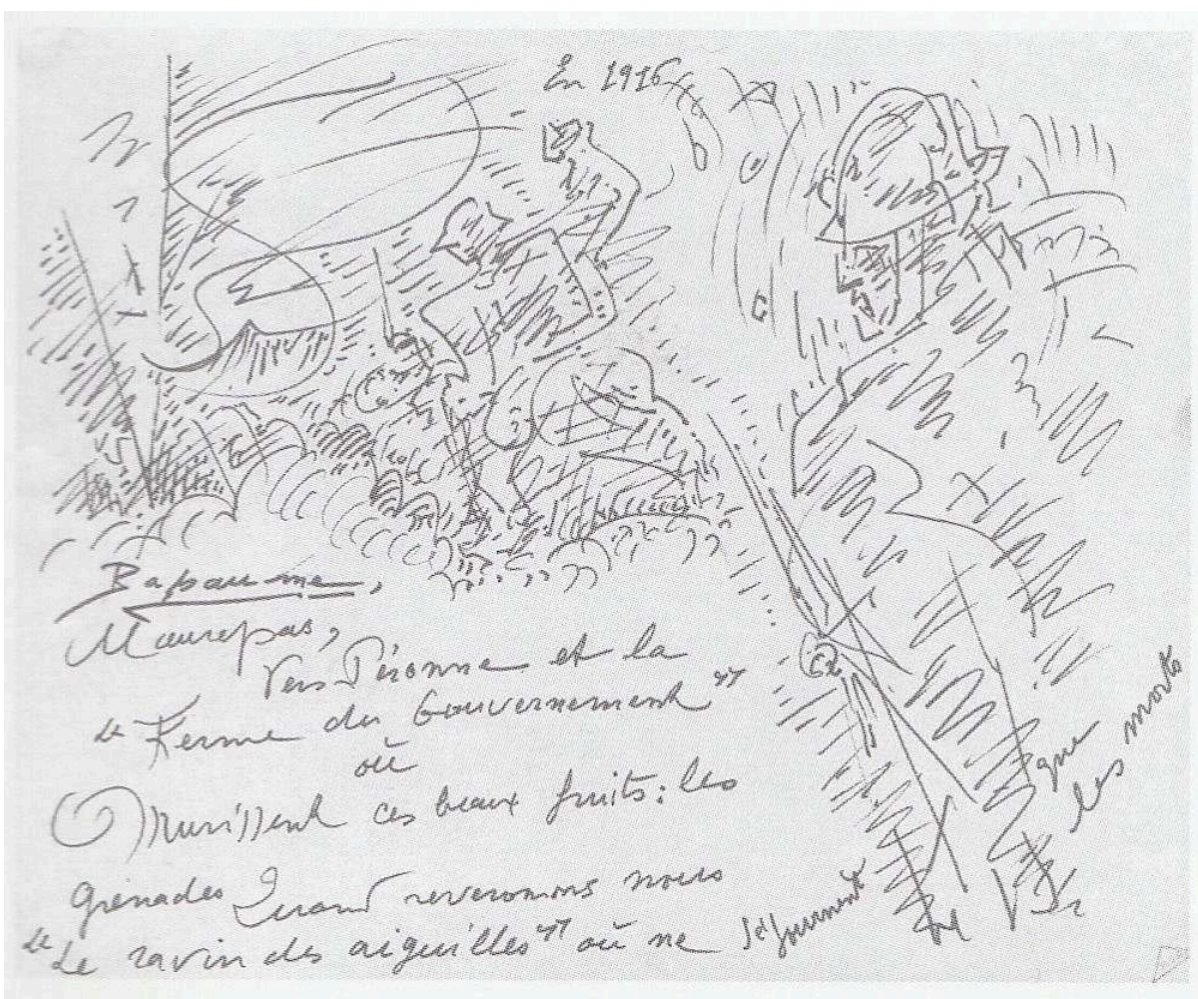

(c)André Masson/Adagp, Paris, 2021

L'organisation des pages et les titres des carnets composés après la guerre varient, bien sûr, selon les exigences des auteurs qui se remémorent leurs expériences combattantes, parfois dans le cadre public de commémorations. En outre, les pages de plus grande taille se révèlent plus propices aux témoignages recomposés, alors que les carnets utilisés au front ont généralement des dimensions plus réduites. Dès lors, comment interpréter ces formats ? Et compte tenu de l'hétérogénéité des sources, quelle information visuelle et quelle iconographie artistique véhiculent-ils ? S'agissant d'objets autobiographiques, une approche socio-historique resitue les carnets dans la trajectoire biographique et selon l'itinéraire géographique et géopolitique de l'artiste combattant. Outre les situations d'inaction (p. ex. troupes au repos) ou les activités très risquées des combats dans les tranchées, il importe également de considérer les autres expériences de la guerre, comme la captivité des soldats dans les camps de prisonniers, les souffrances ou les traumatismes qui très souvent comportent hospitalisations et convalescences. En amont, une herméneutique opératoire consisterait dans le repérage des autoportraits et des signatures qui parfois sont disséminés dans les pages blanches ou dessinées des carnets, mais ce n'est qu'une hypothèse de travail. Par ailleurs, en aval, il ne faut pas négliger le fait que les interruptions, les lacunes ou les blancs constituent autant d'agencements du rythme figuratif que d'espaces de visibilité.

La restitution de l'expérience du front implique les transitions des scénarii et les récursivités iconographiques. Car les déplacements des anciens combattants ne s'effectuent pas seulement entre le front et l'arrière, mais selon une alternance complexe qui peut concerner retours et détours. La médiation du texte s'avère d'autant 
plus significative dans les correspondances ou dans les journaux intimes, permettant aussi bien d'améliorer la réception de la biographie militaire que de rendre plus intelligibles ou lisibles les images. La pratique de l'énumération (p. ex. listes et adresses de contacts) peut concerner titres d'ouvrages ou noms propres, y compris ceux de collègues artistes ou d'intermédiaires. La pratique de la datation atteste en outre l'usage du carnet comme journal intime, ainsi que du besoin d'écrire et de raconter des histoires. En plus de ces enjeux poétiques ou prosaïques, il faut compter enfin les termes techniques et les usages pragmatiques inhérents au service militaire. Dans son histoire sociale de la Grande Guerre, Marc Ferro rappelle que le Maréchal Joffre, au lendemain de la bataille de la Marne, tient un "petit carnet " (105) pour annoter le compte exact des munitions d'artillerie. Dans les carnets d'artistes combattants, il s'agit de construire des tableaux synoptiques (abréviations, schémas, termes techniques), de produire des croquis d'observation (panoramas, engins, uniformes) ou des cartographies du front exécutées sous commande ministérielle ou régimentaire. Les images traduisent les formules standardisées du langage militaire surtout lorsque les artistes combattants intègrent les services à l'arrière du front comme le camouflage militaire ou la cartographie aérienne.

Toutefois, lorsqu'il s'agit de documenter, d'expérimenter ou bien de témoigner, le format du carnet ne convient plus seulement à un usage purement instrumental, mais aussi et surtout à une activité de recherche et de création artistique. Lorsqu'ils en parlent dans leurs récits et mémoires, les maitres ou les élèves des écoles des BeauxArts emploient généralement la notion de pittoresque, alors que les artistes modernes privilégient le discours sur la fragmentation ou sur la métamorphose figurative. À ce propos, André Masson déclare: "C'est qu'il me paraît fatal que certaines épreuves laissent des empreintes dans le laboratoire de la création, celle-ci serait-elle assez puissante pour transmuer en fêtes picturales les massacres, comme le souhaitent Delacroix et Baudelaire » (56). Fatalement, il s'agit d'un morcellement ou bien d'un éparpillement qui caractérise particulièrement le format du carnet de croquis, dont les pages à bords lisses ou segmentés, hier tout comme aujourd'hui, apparaissent suspendues entre la multiplication et la dispersion, la circulation et la conservation, la commande et l'offrande.

\section{Ouvrages cités}

An. « Le 'Salon’ des Armées ». Bulletin des Armées de la République réservé à la zone des Armées 221 (22 novembre 1916) : 7 .

An. « Nos artistes au front ». Lectures pour tous 123 (15 septembre 1916) : 1813-1820.

ARAGON, Louis. « Anicet ou le panorama, roman ». 1921. CEuvres romanesques complètes. Paris : Gallimard, 1997. 1-166. 
BARTRAM, Angela, EL-BIZRI, Nader et Douglas GITTENS (dir.). Recto Verso: Redefining the Sketchbook. Farnham Burlington : Ashgate, 2014.

BONNAT, Léon (dir.). Album national de la guerre. Paris : Bernheim-Jeune \& Cie, 1915.

BULLETIN DES ARMÉES DE LA RÉPUBLIQUE (dir.). Salon des Armées de la République. Catalogue. Paris : Berger-Levrault, 1916.

CAZALS, Rémy et Frédéric ROUSSEAU. 14-18, le cri d'une génération. Toulouse : Privat, 2001.

CLÉMENT-JANIN, Noël. « Les estampes et la guerre (Deuxième article) ». Gazette des Beaux-Arts XIII/4e période (janvier-mars 1917) : 361-383.

CLÉMENT-JANIN, Noël. Les estampes. Images et affiches de la guerre. Paris : Gazette des Beaux-Arts, 1919.

CRU, Jean Norton. Du témoignage. 1930. Paris : Allias, 2008.

DAYOT, Arman. « Un artiste combattant ». L'Illustration 3834 (26 août 1916) : n. p.

DI GENOVA, Giorgio. Storia dell'arte italiana del ‘900. Bologne : Bora, 1993. 1/1.

DIDI-HUBERMAN, Georges. L'Album de l'art à l'époque du « Musée imaginaire ». Paris : Hazan, 2013.

DIDI-HUBERMAN, Georges. « Déplacer voir (Le document, l'archive, l'atlas) ». Critique 879-880

(août-septembre 2020) : 610-624.

DIDIER, Christophe (dir.). Orages de papier, 1914-1918. Les collections de guerre des bibliothèques. Paris : Somogy, 2008.

DIX, Otto, Briefe. Cologne : Wienland, 2013.

FERRO, Marc. La Grande Guerre, 1914-1918. Paris : Gallimard, 1969.

GRAFFIN, Laurence (dir.). André Mare. Carnets de guerre, 1914-1918. Paris : Herscher, 1996.

HEINICH, Nathalie. Faire voir. L'art à l'épreuve de ses médiations. Bruxelles : Les Impressions Nouvelles, 2009.

JUDE, Élisabeth et Patrick JUDE (dir.). Mathurin Méheut. Des ennemis si proches, 1914-1918,. Rennes : Ouest France, 2014.

KEEGAN, John. Anatomie de la bataille. Azincourt 1415, Waterloo 1815, la Somme 1916. 1976. Paris : Robert Laffont, 1993.

LAUXEROIS, Roger (dir.). Adrien Ouvrier. Carnets et croquis de guerre, 1914-1918. Paris : Somogy, 2007.

MARE, André, Dessins faits aux armées. Paris : éditeur inconnu, sans date (1917). <https:// gallica.bnf.fr/ark:/12148/btv1b103159500.item>.

MASSON, André. La mémoire du monde. Genève : Albert Skyra, 1974.

MONTIER, Jean-Pierre. « Constantin Guys selon Baudelaire : reportage et modernité ». Dir. BOUCHARENC, Myriam et Joëlle DELUCHE. Littérature et reportage. Limoges : Presses Universitaires de Limoges et du Limousin, 2000. 187-203.

MUSÉE DE L'ARMÉE ET BIBLIOTHÈQUE DOCUMENTATION INTERNATIONALE CONTEMPORAINE (dir.). Vu du front. Représenter la Grande Guerre. Paris : Somogy, 2014.

NANCY, Jean-Luc. Le Plaisir au dessin. Paris : Galilée, 2009.

OTTINGER, Didier (dir.). Max Beckmann. Gravures, 1911-1946. Paris : Réunion des Musées Nationaux, 1994. 
ROUVEYRE, Édouard. Connaissances nécessaires à un bibliophile. Paris : Librairie ancienne et moderne, 1879. <https://gallica.bnf.fr/ark:/12148/bpt6k64898191.texteImage>.

STRUK, Janina. Private Pictures: Soldiers' Inside View of War. New York : Tauris, 2011.

TOMASEK, Michel (dir.). Joseph Dezitter, 1883-1957. Artiste flamand. Dunkerque : Société dunkerquoise d'histoire et d'archéologie, 2012.

TONOLLI, Frédéric, réal. André Mare, carnets de guerre d'un caméléon. France 5, 2014.

VACHÉ, Jacques. Soixante-dix-neuflettres de guerre. Paris : Jean-Michel Place, 1989.

VATIN, Philippe. Voir et montrer la guerre. Images et discours d'artistes en France, 1914-1918. Dijon : Les Presses du Réel, 2013.

ZAPATA-AUBÉ, Nicole (dir.). André Mare. Cubisme et camouflage. Bernay : Musée des Beaux-Arts, 1998.

ZEILLER, Christiane (dir.). Max Beckmann. Die Skizzenbücher. Munich : Hatje Cantz, 2010.

\section{NOTES}

1. Dans son analyse critique du témoignage combattant, l'écrivain français Jean Norton Cru adopte justement un cadre large incluant non seulement les soldats d'infanterie ou d'artillerie, mais aussi les membres des services sanitaires du front, certains soldats du Génie ou de la Territoriale : « La guerre elle-même a imposé cette définition fondée sur l'exposition au danger et non plus sur le port des armes qui ne signifie plus rien » (24). Dans le domaine de l'histoire militaire, John Keegan s'est intéressé particulièrement aux interactions complexes parmi les troupes combattantes dans la bataille de la Somme (1916).

2. Voir les collections numérisées de la bibliothèque de l'Institut de France et de la bibliothèque numérique de l'Institut National d'Histoire de l'Art (INHA) : http:// leonardcarnets.institutdefrance.fr ; https://bibliotheque-numerique.inha.fr/ collection/item/6287-eugene-delacroix-carnet-heliotrope (pages consultées le 5 mai 2021).

3. Les contributions de Philippe Vatin s'avèrent parmi les plus significatives dans le domaine de l'iconographie artistique, bien que l'auteur se limite à traiter les carnets à l'aune des pratiques discursives d'artistes français : «la guerre semble un moment propice à la transparence des fonctions graphiques » (19).

4. Voir également la base de données collaborative et encyclopédique « Carnets de guerre ", mise en ligne par quatre archives départementales françaises (AlpesMaritimes, Indre-et-Loire, Pas-de-Calais et Saône-et-Loire) : https://fr.wikisource.org/ wiki/Wikisource:Carnets_de_guerre (page consultée le 5 mai 2021).

5. https://portail-collections.imec-archives.com/exhibit/946 (page consultée le 5 mai 2021).

6. https://argonnaute.parisnanterre.fr/exhibit/13 (page consultée le 5 mai 2021).

7. https://argonnaute.parisnanterre.fr/ark:/14707/a011403268236JL4Gvz (page consultée le 5 mai 2021). 
8. Voir le document accessible en ligne dans la bibliothèque numérique « L'Argonnaute » de La Contemporaine : https://argonnaute.parisnanterre.fr/medias/customer_3/ photos/camus_or_9356/BDIC_OR_009356_192_1.jpg (page consultée le 5 mai 2021).

9. Albano Vitturi, Album de guerre. 1922. Huile sur toile, 75x80 cm. Coll. privée.

10. https://gallica.bnf.fr/ark:/12148/btv1b103159500.item (page consultée le 5 mai 2021).

11. Voir aussi l'exposition virtuelle des fonds numérisés des archives de la Cité de l'architecture et du patrimoine ( Un architecte dans la Grande Guerre : album de croquis de Jacques Carlu ») : https://expositions-virtuelles.citedelarchitecture.fr/EXPOVIRTUELLE-CARLU/00-OUVERTURE.html (page consultée le 5 mai 2021).

12. Dans le domaine de la fabrication manuelle ou mécanique du papier, le terme pontuseau désigne généralement « chacune des lignes claires, verticales et espacées visibles par transparence dans la feuille de papier à la forme qui coupe perpendiculairement les vergeures ». https://www.cnrtl.fr/definition/pontuseau (page consultée le 5 mai 2021).

13. Trad. personnelle de l'allemand.

14. Dans La mémoire du monde (1974), Masson reproduit ses dessins de guerre dans un « Carnet de route " non paginé (Musée de l'Armée et Bibliothèque Documentation Internationale Contemporaine 353-55). Le "Carnet de guerre " édité posthume de Dezitter (2012) s'intitule « Impressions de campagnes et de voyages, 1914-1915-1916 » (Tomasek 136-73). Il s'agit également d'un témoignage recomposé.

\section{RÉSUMÉS}

Les carnets d'artistes combattants ont fait l'objet d'éditions, d'expositions et ventes, ainsi que de numérisations et d'expositions virtuelles dans le cadre des missions pédagogiques du centenaire de la Grande Guerre. Cet article étudie les enjeux philologiques et patrimoniaux du carnet de guerre en explorant plusieurs contextes et domaines: archives et collections, expositions, éditions et presse illustrée. Les formats des carnets d'artistes combattants nous invitent à confronter genres et thèmes iconographiques, pratiques artistiques et littéraires, profils et trajectoires biographiques. Du fait que ces carnets contiennent notations graphiques, chromatiques, textes et photographies, les recherches et les montages documentaires sont confrontés à l'hétérogénéité et à l'hybridité des formats, tandis que leurs usages vont de l'expérimentation artistique au projet éditorial.

The sketchbooks of combatant artists have been the objects of editions, exhibitions, sales, as well as digitization and virtual exhibitions in the context of the educational projects of the centenary of the Great War. This article studies the philological and heritage issues of the war sketchbook, exploring a variety of contexts and fields: archives and collections, exhibitions, book editions and the illustrated press. Studying the format of the combatant artists' sketchbooks implies the comparison between iconographic genres and themes, artistic and literary practices, biographical profiles and trajectories. As those sketchbooks contain graphic and chromatic notations, texts and photos, documentary research and montage have to deal with the 
heterogeneity and the hybridity of their formats, while the practices involved range from artistic experimentation to editorial projects.

\section{INDEX}

Mots-clés : album, artiste combattant, carnet de guerre, édition d'art, études visuelles, exposition virtuelle, Grande Guerre, humanités numériques, philologie, témoin oculaire Keywords : album, combatant artist, war sketchbook, art edition, visual studies, virtual exhibition, Great War, digital humanities, philology, eyewitness

\section{AUTEUR}

\section{MARCO FALCERI}

CRISES (EA 4424 Centre de recherches interdisciplinaires en sciences humaines et sociales) et UPVM3 (Université Paul-Valéry Montpellier 3)

Marco Falceri est doctorant en histoire contemporaine à l'Université Paul-Valéry Montpellier 3, où il réalise une thèse intitulée « Les artistes combattants de la Grande Guerre : une histoire des arts visuels ». Privilégiant une approche pluridisciplinaire de l'iconographie des guerres modernes et contemporaines, ses recherches portent sur les usages poétiques et politiques du témoignage combattant. Parmi ses contributions spécifiques : « Arts visuels et traumatisme dans la Grande Guerre : témoigner la vulnérabilité », Doctorales-58 5 (2018). 